\title{
Two Staged Portfolio Optimizations
}

Hamid Ahmadi, (Email: ahmadih@csus.edu), California State University, Sacramento Cesar Galindo, (Email: famolare-95205@yahoo.com), California State University, Sacramento

\begin{abstract}
The primary goal of this work is to determine if an active portfolio optimization strategy utilizing a two staged optimization approach outperforms an ordinary optimization technique. Both portfolio optimization models are based on Markowitz's Modern Portfolio Theory (MPT), which relies on assets' mean, variance, and correlation to maximize returns at any given level of risk.
\end{abstract}

For the two staged optimization approach the process of optimization is applied twice. In the first stage, it is used to select an optimal portfolio of industries, and in the second stage optimization is applied to determine an optimal portfolio consisting of stocks within each industry.

Our research indicates that portfolios formed based on ordinary optimization outperforms two staged portfolios and Market indexes by 37\% during a bear market (2002) and outperforms Dow Jones Industrial Average and S\&P 500 by more than 13\% during a bull market (2003). The performance of each model was determined by the capital gains and the dividend returns during the 2002 to 2003 time period.

\section{INTRODUCTION}

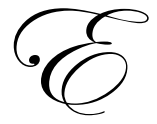

ver since Markowitz introduced the mean-variance optimization (MVO) process in 1952, numerous books, articles, and discussion papers have been published on the formation of the efficient portfolios using Markowitz's Modern Portfolio Theory (MPT). Furthermore, over the years researchers fine-tuned the model and developed complex, innovative and yet more precise portfolio optimization techniques which are all based on Markowitz's original work.

Although few of the investment strategies are specialized and include complex derivatives, a common investment strategy typically requires an investor to diversify among asset class such as bonds, real estate, and equity. More experienced investors, however, pursue broader investment approaches by incorporating wide range of asset classes such as municipal bonds, corporate bonds, commercial real estates, small, mid and large capitalization stocks. The larger investment firms, on the other hand, support portfolio managers to utilize the expertise of the registered specific-asset specialists and include rare investment opportunities in U.S., Europe, and emerging markets.

Numerous investment approaches also exist solely on the aspects of rebalancing. Current practices range from chaotic to disciplined rebalancing. Some portfolio managers rebalance their portfolios when the cash inflows or outflows dictate. Others base their rebalancing strategies on times such as monthly, quarterly or semiannually and others base it on the deviation from target allocations such as one, five or ten percent (Buetow, et al., 2002). According to Buetow, et al., (2002), the primary criteria for rebalancing a portfolio is based on reducing risks. Buetow, et al., (2002, p.1) states "portfolios that are not rebalanced can loose some of these risk reduction benefits, drifting toward an unintended large percentage of higher-risk assets or inefficient allocations."

Other investment strategies rely on proprietorship stocks rating systems to construct a model. Value Line manages three types of portfolio models constructed from 20 stocks based on its ratings system and tailored to an investor's risk preference (aggressive, moderate, and long-term) (Valueline, 2004). MSN Moneycentral recommends building a 50 stock portfolio with monthly balances to fully take advantage of its StockScouter system (MSN Moneycentral, 2004). Similar to Value Line, portfolios can be tailored to an investor's risk preference. However, the 
StockScouter system allows for even greater customization such as favoring value stocks, large-cap stocks, high-rated corporate stocks and so forth.

For those portfolio optimization strategies employing an MVO technique, there also exist numerous variations of MVO techniques. The most popular MVO technique is the Minimum Variance Portfolio (MVP) which is essentially the portfolio on the efficient frontier curve that offers the maximum possible expected return for the minimum level of risk. Some other MVO techniques discussed in recent literature are the certainty-equivalencetangency (CET) and the Bayes-Stein Estimation Method, which is also know as the Bayes-Stein Transformation (BST). In these models Larsen and Resnick (2001) incorporate a shrinkage factor into the expected excess return equation which allows them to place more weight on the smaller size-based portfolios.

\section{PURPOSE}

The primary goal of this work is to determine if an active portfolio optimization strategy utilizing a two staged optimization approach outperforms an ordinary optimization technique. Both portfolio optimization models are based on Markowitz's Modern Portfolio Theory (MPT), which relies on assets' mean, variance, and correlation to maximize returns at any given level of risk.

For the two staged optimization approach the process of optimization is applied twice. In the first stage, it is used to select an optimal portfolio of industries, and in the second stage optimization is applied to determine an optimal portfolio of stocks within each industry. For the ordinary optimization approach the process of optimization is applied once. It is used to select an optimal portfolio of stocks within the stock market.

Lagrange Constraint Optimization (LCO) is used as the MVO technique to generate the portfolio that offers the maximum possible expected return for any given level of risk. The distinguishing characteristic of the LCO technique is that it minimizes the variance of the portfolio subject to two or more constraints. These constraint capabilities were used to constrain each asset to a minimum value of zero percent, in other words, no shorting of a stock was allowed. The rational behind disallowing shorts is that it is seldom practiced in the real world due to the risk of unlimited losses. Furthermore, preliminary analysis revealed portfolios that took into account shorting, drastically underperformed the Market index. An active investment strategy was chosen to reduce the risk of moving away from client's expectations or wrong allocations over time.

All dividends returns for each investment cycle were reinvested biweekly. The performance of each model was determined by the capital gains and the dividend returns during the 2002 to 2003 time period.

\section{THEORY OF PORTFOLIO OPTIMIZATION}

\section{Lagrange Constraint Optimization For 3 Asset Portfolio}

The Lagrangean approach is used to minimize the variance of the portfolio subject to two constraints. This is accomplished by taking the first derivative of the Lagrangean function and equating them to zero. The simultaneous linear functions are presented in a matrix format known as the Border Hessian Matrix (BHM). The main advantage for presenting them in matrix form is the ease and speed in which one can solve for the variables using matrix algebra. The following section provides an expanded view of a six step process for Lagrange Constraint Optimization with the BHM for a three asset portfolio. 


\section{Optimization with two Constraints:}

$$
\begin{aligned}
& \text { Min: } \quad \text { Var }(\mathrm{P}) \\
& \text { Subject to: } \quad\left\{\begin{array}{l}
x_{1}+x_{2}+x_{3}=1 \\
x_{1} k_{1}+x_{2} k_{2}+x_{3} k_{3}=R p
\end{array}\right.
\end{aligned}
$$

Where $\mathrm{X}_{\mathrm{i}}$ is the proportion invested in asset $\mathrm{i}$, and $\mathrm{K}_{\mathrm{i}}$ is the average return of asset $\mathrm{i}$; and $\operatorname{Var}(\mathrm{p})$ is the variance of the portfolio.

\section{Lagrangean Function:}

$$
\begin{aligned}
& \left.L=f()-\lambda_{1}\left(1^{\text {st }} \text { constra int }\right)-\lambda_{2}\left(2^{\text {nd }} \text { constra int }\right)\right) \\
& L=\operatorname{var}(p)-\lambda_{1}\left(x_{1}+x_{2}+x_{3}-1\right)-\lambda_{2}\left(x_{1} k_{1}+x_{2} k_{2}+x_{3} k_{3}-R p\right) \\
& L=x_{1}^{2} \operatorname{var}(1)+x_{2}^{2} \operatorname{var}(2)+x_{3}^{2} \operatorname{var}(3)+2 x_{1} x_{2} \operatorname{cov}(1,2)+2 x_{1} x_{3} \operatorname{cov}(1,3)+2 x_{2} x_{3} \operatorname{cov}(2,3) \\
& \underbrace{-\lambda_{1}\left(x_{1}+x_{2}+x_{3}\right.}_{-\lambda_{1} x_{1}-\lambda_{2} x_{2}-\lambda_{3} x_{3}}-1) \underbrace{-\lambda_{2}\left(x_{1} k_{1}+x_{2} k_{2}+x_{3} k_{3}\right.}_{-\lambda_{1} x_{1} k_{1}-\lambda_{2} x_{2} k_{2}-\lambda_{3} x_{3} k_{3}}-R p)
\end{aligned}
$$

$\mathrm{L}$ is the Lagrangean function, and $\lambda_{1}$ and $\lambda_{2}$ are Lagrangean multipliers.

\section{1st Derivative of Lagrange Function:}

$$
\begin{aligned}
& \frac{d L}{d x_{1}}=2 x_{1} \operatorname{var}(1)+2 x_{2} \operatorname{cov}(1,2)+2 x_{3} \operatorname{cov}(1,3)-\lambda_{1}-\lambda_{2} k_{1}=0 \\
& \frac{d L}{d x_{2}}=2 x_{2} \operatorname{var}(2)+2 x_{1} \operatorname{cov}(1,2)+2 x_{3} \operatorname{cov}(2,3)-\lambda_{1}-\lambda_{2} k_{2}=0 \\
& \frac{d L}{d x_{3}}=2 x_{3} \operatorname{var}(3)+2 x_{1} \operatorname{cov}(1,3)+2 x_{2} \operatorname{cov}(2,3)-\lambda_{1}-\lambda_{2} k_{3}=0 \\
& \frac{d L}{d \lambda_{1}}=-\left(x_{1}+x_{2}+x_{3}-1\right)=0 \\
& \frac{d L}{d \lambda_{2}}=-\left(x_{1} k_{1}+x_{2} k_{2}+x_{3} k_{3}-R p\right)=0
\end{aligned}
$$

\section{Rearranging the linear equations:}

$$
\begin{aligned}
& 2 x_{1} \operatorname{var}(1)+2 x_{2} \operatorname{cov}(1,2)+2 x_{3} \operatorname{cov}(1,3)-\lambda_{1}-\lambda_{2} k_{1}=0 \\
& 2 x_{1} \operatorname{cov}(1,2)+2 x_{2} \operatorname{var}(2)+2 x_{3} \operatorname{cov}(2,3)-\lambda_{1}-\lambda_{2} k_{2}=0 \\
& 2 x_{3} \operatorname{var}(3)+2 x_{1} \operatorname{cov}(1,3)+2 x_{2} \operatorname{cov}(2,3)-\lambda_{1}-\lambda_{2} k_{3}=0 \\
& x_{1}+x_{2}+x_{3}-=1 \\
& x_{1} k_{1}+x_{2} k_{2}+x_{3} k_{3}=R p
\end{aligned}
$$




\section{System of linear equations:}

After rearranging the first derivative equations, the above system of equations can be written in matrix form.

$$
\begin{array}{ccccc}
{\left[\begin{array}{ccccc}
2 \operatorname{var}(1) & 2 \operatorname{cov}(1,2) & 2 \operatorname{cov}(1,3) & 1 & k_{1} \\
2 \operatorname{cov}(1,2) & 2 \operatorname{var}(2) & 2 \operatorname{cov}(2,3) & 1 & k_{2} \\
2 \operatorname{cov}(1,3) & 2 \operatorname{cov}(2,3) & 2 \operatorname{var}(3) & 1 & k_{3} \\
1 & 1 & 1 & 0 & 0 \\
k_{1} & k_{2} & k_{3} & 0 & 0
\end{array}\right]\left[\begin{array}{c}
x_{1} \\
x_{2} \\
x_{3} \\
-\lambda_{1} \\
-\lambda_{2}
\end{array}\right]=\left[\begin{array}{c}
0 \\
0 \\
0 \\
1 \\
R p
\end{array}\right]} \\
{[\mathbf{B H M}]} & {[\mathbf{X}]=[\mathbf{C}]}
\end{array}
$$

\section{Critical Line Equation}

The critical line is the line which connects all the efficient points (the points with the highest possible return for a given risk). From the critical line equation the proportions of the assets $\left(\mathrm{X}_{1}, \mathrm{X}_{2}\right.$, and $\left.\mathrm{X}_{3}\right)$ can be computed for any given portfolio return (Rp). The critical line equation for each asset in the portfolio is determined using the following matrix equations:

$$
\begin{gathered}
{[\mathrm{BHM}][\mathrm{X}]=[\mathrm{C}]} \\
{[\mathrm{X}]=\left[\mathrm{BHM}^{-1}\right][\mathrm{C}]} \\
{\left[\begin{array}{c}
x_{1} \\
x_{2} \\
x_{3} \\
-\lambda_{1} \\
-\lambda_{2}
\end{array}\right]=\left[\text { BHM }^{-1}\right]\left[\begin{array}{c}
0 \\
0 \\
0 \\
1 \\
R p
\end{array}\right]}
\end{gathered}
$$

\section{SIMPLIFICATIONS}

In order to simplify the workload required on this project, several crucial simplifications had to be used. The first crucial simplification allowed for the generation of monthly returns for varying monthly rebalance dates. It essentially follows what is done in practice in order to generate the monthly returns, that is to roll back the date to the same day in the previous month and sum up the returns in this new date range.

Further simplification involved selecting Friday as the rebalance day for each portfolio. If the market happened to be closed on Friday, then Thursday was selected.

Another simplification entailed using the coefficient of variation (CV) as the key attribute in determining an asset's relative risk prior to constructing portfolios. CV measures relative risk for each asset $(C V=|\sigma / \bar{X}|=$ Standard Deviation / Average Return).

This last crucial simplification only applies to the two staged optimization approach. Its intent is to not exclude unpopular industries such as jewelry stores, music and video stores, sporting activities, and so on. The thinking here is that there is usually at least one or two dominant (large) company(s) within any industry that 
outperforms the rest. Thus, any unpopular industry can be represented with lesser risk by selecting the most dominant company(s) within the industry.

\section{TWO STAGED OPTIMIZATION APPROACH}

As mentioned before, the process of optimization is applied twice in the two staged optimization approach. In the first stage, it is used to select an optimal portfolio of industries, and in the second stage optimization is applied to determine an optimal portfolio of stocks within each industry. However, before any optimization could take place in the first stage, an optimal number of industries had to be determined.

The optimal number of industries was determined by finding the point of decreasing maximum slope on the efficient frontier curve while varying the number of assets in the portfolio. The highest performing portfolios required roughly 10 or more assets to eventually hit their crest. However, to reduce the complexity of computing, nine assets were chosen as the number of industries.

The stock selection process for the two staged optimization approach consists of three core phases. In the first phase, all the raw data required for the stock selection process is retrieved and prepared for processing. The goal of the second phase is to identify the 15 most promising low risk stocks based on our criteria. The criteria consisted of market capitalization, averaged daily volume, $\mathrm{P} / \mathrm{E}$ ratio, and $\mathrm{CV}$.

The intent of the final selection process is to generate a three stock portfolio for each industry optimized with LCO. A target of three stocks per industry was selected because it provides roughly 30 stocks per rebalance period. This allows for better comparisons to the ordinary optimization technique which averages roughly 20 to 30 stocks per rebalance period. Furthermore, each portfolio was optimized using 29 to 36 monthly data points for each stock.

There are numerous resources available for ratings stocks. Some of the more popular ratings used by investors consist of Value Line and MSN Moneycentral Stockscouter to name a few.

\section{ORDINARY OPTIMIZATION TECHNIQUE}

As mentioned before, the process of optimization is applied once in the ordinary optimization approach. In this approach it is used to select an optimal portfolio of stocks within the stock market. The ordinary optimization technique consists of three core phases. In the first phase, all the raw data required for the stock selection process is retrieved and prepared for processing. The goal of the second phase is to identify 60 to 80 of the most promising low risk stocks based on a stocks average daily volume and CV. The final phase optimized with the LCO technique produced roughly 20 to 30 stocks for investment.

\section{RESULTS OF ORDINARY AND TWO STAGED OPTIMIZATION}

The performance of each strategy was evaluated primarily based on how its returns performed against each other and the two key Market indexes, Dow Jones Industrial Average and S\&P 500. All dividends returns for each investment cycle were reinvested in the following rebalance period. The dividends and capital gains were used in determining the performance (returns) of each portfolio from one rebalance period to the next. In addition, each strategy's performance was evaluated during 2002 and 2003 using U.S. Equity historical data.

A biweekly investment approach provided a total of 26 investment periods per year. Each investment period incorporated buys, sells, and rebalances (decrease or increase investments of current assets) as the models recommended. In 2003, the two staged model contained 756 transactions whereas the ordinary model contained 523. This equates to a $46 \%$ increase in the number of transactions switching from an ordinary optimization technique to a two staged optimization approach. As one can incur, transaction costs could play a large factor in determining a 
model's performance. However, in this analysis the impact of transaction cost on a portfolio's return was considered insignificant.

In 2002, the ordinary optimization technique significantly outperformed the two staged optimization approach by over $51 \%$. In addition, it significantly outperformed both the DJIA and the S\&P500 by over 52\%. In 2003 , the ordinary optimization technique moderately outperformed the two staged optimization approach by $7 \%$ and both the DJIA and the S\&P 500 by over $14 \%$ (see table 1). Even more, the ordinary optimization techniques provides a smaller standard deviation of return compared to the two staged optimization approach (see table 2). Based on these findings, one can conclude that the ordinary optimization technique is the more optimal and robust than the two staged optimization approach.

Understanding exactly why the ordinary optimization technique performed better than the two staged optimization approach is the subject of further studies. One can infer that because the two staged optimization process focuses only on stocks found within a few industries, it neglects more promising stocks found throughout the stock market. The ordinary investment approach on the other hand scours the entire stock market for the most promising stocks. Furthermore, one can also infer that because the two staged optimization strategy breaks down its investments into nine smaller distinct portfolios, fewer stocks are used in correlating amongst each other. The ordinary investment technique pools all its stocks together into one larger portfolio. This allows all its stocks to be correlated amongst each other, thereby profiting more from MVO.

Table 1: Summary of Portfolio Model Returns

\begin{tabular}{|c|c|c|c|c|c|}
\hline & 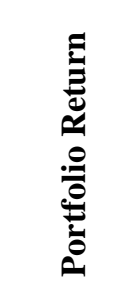 & 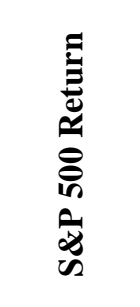 &  &  & 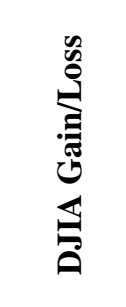 \\
\hline \multicolumn{6}{|c|}{2002} \\
\hline Ordinary Strategy & $\mathbf{3 4 . 8 1 \%}$ & $-24.60 \%$ & $-18.08 \%$ & $59.41 \%$ & $\mathbf{5 2 . 8 9 \%}$ \\
\hline Two Staged Strategy & $-16.90 \%$ & $-24.60 \%$ & $-18.08 \%$ & $7.70 \%$ & $1.18 \%$ \\
\hline \multicolumn{6}{|c|}{2003} \\
\hline Ordinary Strategy & $41.94 \%$ & $20.56 \%$ & $19.95 \%$ & $19.95 \%$ & $21.99 \%$ \\
\hline Two Staged Strategy & $34.99 \%$ & $20.56 \%$ & $19.95 \%$ & $14.44 \%$ & $15.04 \%$ \\
\hline
\end{tabular}

Table 2: Portfolio Investment Characteristics

\begin{tabular}{|c|c|c|c|c|}
\hline \multirow{2}{*}{$\begin{array}{c}\text { Investment } \\
\text { Approach }\end{array}$} & \multicolumn{2}{|c|}{$\begin{array}{c}\text { Average \# of Stocks / } \\
\text { Investment Period }\end{array}$} & $\begin{array}{c}\text { Standard Deviation of } \\
\text { Weekly Portfolio } \\
\text { Performance }\end{array}$ \\
\cline { 2 - 5 } & $\mathbf{2 0 0 2}$ & $\mathbf{2 0 0 3}$ & $\mathbf{2 0 0 2}$ & $\mathbf{2 0 0 3}$ \\
\hline \hline Ordinary & 23 & 19 & $3.83 \%$ & $2.11 \%$ \\
\hline Two Staged & 33 & 28 & $3.51 \%$ & $4.12 \%$ \\
\hline
\end{tabular}


It is important to note that there is a significant change in the performance of the ordinary optimization technique compare to the two staged optimization depending on the year. That is, in 2002, the ordinary optimization technique outperformed the two staged optimization approach by a staggering 51.7\% whereas in 2003, it outperformed it by only 7\%. The most notable difference between 2002 and 2003 data is that 2002 is considered bear (down) market whereas 2003 is considered a bull (up) market. Exactly why the ordinary optimization technique performs so much better than two staged optimization approach during a bear as compared to a bull market is the subject to further studies.

Based on the findings, it is possible for one to profit from diversification and market momentum using an active ordinary portfolio optimization technique. However, more research and analysis is required to determine a robust optimal model that outperforms irrespective of the stock market conditions.

Due to the countless number of elements that affect the outcome of a portfolio performance, many key elements had to be simplified or eliminated for this analysis. Brief summaries of several major factors that affect a portfolio optimization model's performance and recommendations for future studies are discussed in the appendix. Ultimately, the goal of these additional analyses is to develop a more optimal model that outperforms irrespective of stock market conditions.

Figure 1: Ordinary vs. Two Staged 2002 Cumulative Returns




Figure 2: Ordinary vs. Two Staged 2003 Cumulative Returns

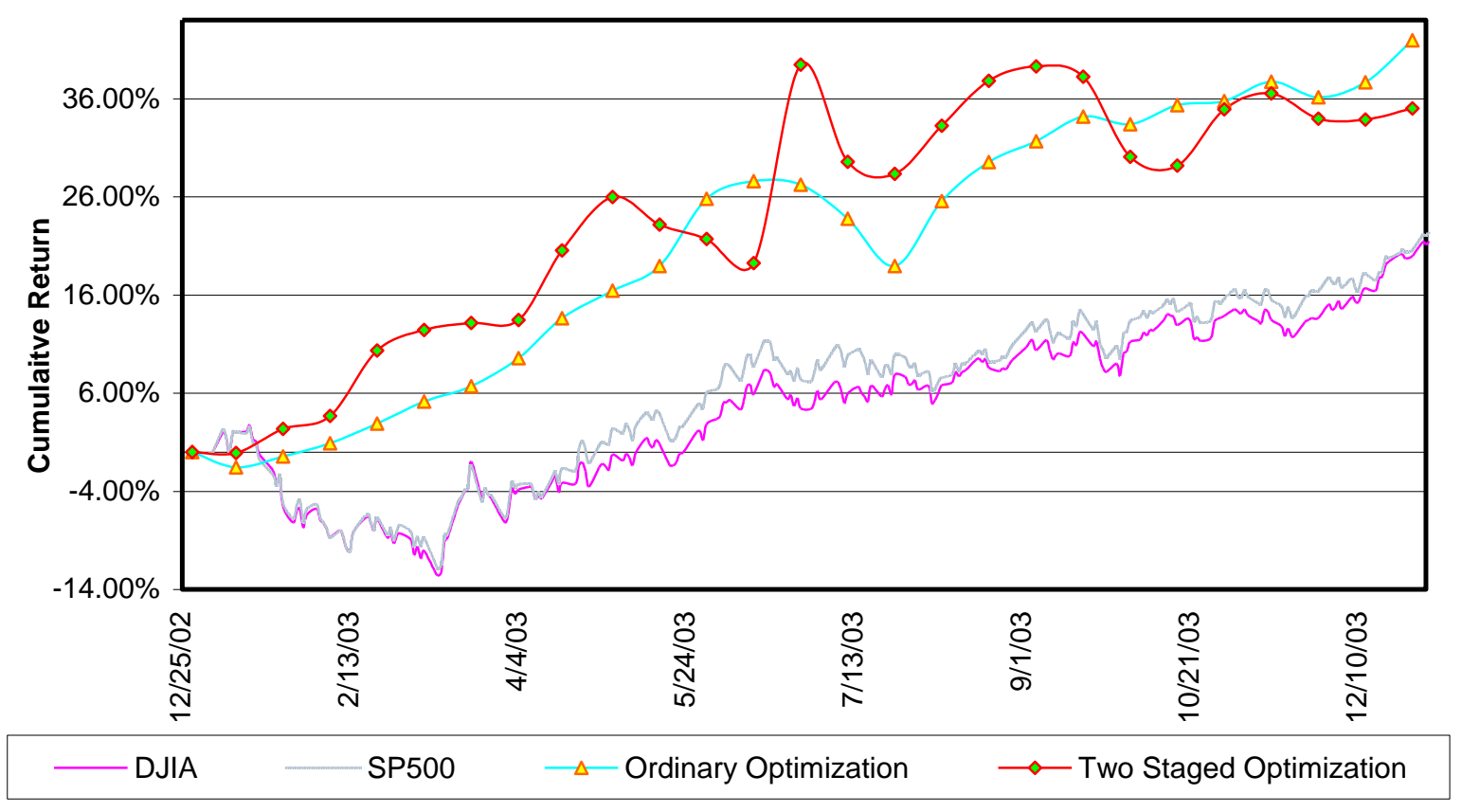

\section{BIBLIOGRAPHY}

1. Ahmadi, H. \& Soriano B. C. (2003). "An Analytical Technique for Determining Efficient Portfolios". American Academy of Accounting and Finance, Proceedings.

2. Ahmadi, H. (1996). "Testability of the Arbitrage Pricing Theory by Neural Networks". Neural Networks in Finance and Invest. Chicago: Probus Publishing Company.

3. Bernstein, W. J. (2001). The Intelligent Asset Allocator - How to Build Your Portfolio to Maximize Returns and Minimize Risk ( $1^{\text {st }}$ ed.). New York: McGraw-Hill.

4. $\quad$ Benninga, S. (2001). Financial Modeling (2nd ed.). Massachusetts: The MIT Press, Cambridge.

5. Buetow Jr., G. W., Sellers, R., Trotter, D., Hunt, E., \& Whipple Jr., W. A. (2002). "The benefits of rebalancing: Worth the effort". Journal of Portfolio Management, 28, 23-39.

6. Clarke, R. G., de Silva, H., \& Wander, B. (2002). "'Risk allocation versus asset allocation: improving the optimal allocation between risk and return". Journal of Portfolio Management, 29, 9-22.

7. Jorion, P. (1986). "Bayes-Stein Estimation for Portfolio Analysis". Journal of Financial and Quantitative Analysis, 21, 279-292.

8. Kaplan, P. D. (1998). “Asset Allocation Using Markowitz Approach”. Retrieved March, 13, 2004, from http://www.ibbotson.com/content/kc_published_research_search.asp?catalog=Article\&category=All\%20 Articles\&prodID=ARTC41020029

9. Larsen, G. A., \& Resnick, B. G. (2001). "Parameter Estimation Techniques, Optimization Frequency, and Portfolio Return Enhancement". Journal of Portfolio Management, 27, 27-35.

10. Lummer, S. L., Riepe, M. W., \& Siegal, L. B. (1994). "Taming Your Optimizer: A Guide through the Pitfalls of Mean-Variance Optimization". Retrieved June 11, 2004, from http://www.ibbotson.com/content/kc_ published_research_search.asp?catalog=Article\&category=Asset\%20Allocation\&prodID=ARTC41120024

11. Madey, R. F., Mulvey, J. M., \& Pauling, W. R. (2003). "Advantages of multi-period portfolio models: performance enhancements". Journal of Portfolio Management, 29, 35-46.

12. Markman, J. (2004). "StockScouter rates potential reward, and risk". Retrieved February, 18, 2004 from http://moneycentral.msn.com/content/investing/findhotstocks/p38814.asp 
13. "Model Portfolios". (n.d.) Retrieved February, 18, 2004, from http://www.valueline.com/ secure/vlispdf/ stk1700/selected_intro.html

14. "Portfolio Theory". (1996). Retrieved February, 18, 2004, from http://www.riskglossary.com/articles/ portfolio_theory.htm\#Modern\%20portfolio\%20theory

15. "Solver Tutorial for Optimization Users" (n.d.). Retrieved March 13, 2004, from http://www.solver.com/ tutorial.htm

16. "The Sharpe Ratio". (n.d.). Retrieved April, 1, 2004, from http://www.moneychimp.com/articles/risk/ sharpe_ratio.htm

\section{APPENDIX - QUALIFICATIONS}

\section{Input Sensitivity Limitation}

One of the limitations of the mean-variance optimization process is the sensitivity of mean-variance model recommendations to input assumptions. "A famous example involves the risk-tolerant end of the efficient frontier. Given two assets with essentially the same projected expected values (e.g. 10\%) and variances (e.g. 20\%), the MV model will arbitrarily pick one of these assets and put $100 \%$ of the investor's money into that asset, while essentially ignoring the other one." (Mulvey, et al., 2003, p.1). Most portfolio managers understand this deficiency and address it by placing fix limits on the proportion of total assets they invest in any single asset category or security (Mulvey, et al., 2003). In terms of sensitivity of the mean-variance model recommendations to input assumptions, no restrictions are placed on the models used for this work. It is not uncommon to occasionally find 70 to 90 percent of funds invested in a single asset as recommended by the models. Further analysis could entail incorporating such limitations.

Another limitation of mean-variance analysis is that its results can sometimes be unstable. According to Kaplan (1998, p.9), "a small move along the efficient frontier could correspond to a large change in asset allocation (which generally should not be implemented). This kind of instability happens when the correlation matrix is illconditioned. The most common cause of the correlation matrix being ill-conditioned is the inclusion of very similar assets in the analysis." Given that all portfolios in this work were constructed solely from U.S. equities, it is likely that some instability is present within the optimal portfolios generated, especially when compared to market portfolios. Other than frequent rebalancing frequencies and the stated filtering processes, no additional steps were taken to diversify among the U.S. equities selected beyond what the optimized portfolio model suggested. Future studies could involve constructing portfolios consisting of other assets such as bonds and international equity besides just U.S. equity.

\section{The Filtering Of Lucrative Companies}

One of the main challenges of the stock selection process involved the elimination of highly rated mid to large companies because they did not meet the minimum 29 monthly data points' requirements. The choice was made to filter them out because it would invalidate the underlying statistical assumptions which are the key elements in the optimizing process. A supplementary study could consider devising a method to incorporate these highly rated mid to large companies that did not meet the minimum data point requirements for MVO.

\section{Survivorship Bias}

This work retrieves past data for companies that currently exist on MSN Moneycentral, which are the survivors up to May 2004. Companies that have become de-listed for whatever reasons such bankruptcies, buy outs, mergers and so forth have automatically been filtered from the stock and industry selection process. What impact or how great of an impact this has on the outcomes of the optimal portfolio models analyzed in real time verses those analyzed presently is unknown. One may imply that the results are skewed in favor of the optimal portfolio models analyzed during the present because of the survivorship bias. Future studies may want to consider incorporating nonsurvivor companies to ensure more realistic results. 


\section{Tax Implications}

Given that all portfolio models are rebalanced a biweekly period, the effect of taxes do no play a role in determining the most optimal model. It would be a different story if a portfolio was rebalanced on a yearly basis because then it could benefit from the long-term tax rates. Further studies could investigate the impact taxes have on active portfolios with greater depth.

\section{Transaction Cost Implications}

Depending on the number of transactions, cost per transaction, and dollar amount associated with a particular portfolio model, its return could vary significantly. For instance, a transaction cost of $\$ 10$ on a $\$ 1,000,000$ portfolio with 1,000 yearly transactions reduces that portfolio's total yearly return by $1 \%$. A $\$ 500,000$ portfolio with the same conditions would have its total return reduced by $5 \%$, which is quite significant. An auxiliary work could take into account transaction cost, however insignificant or significant it might be.

\section{P/E Ratio}

For the two staged optimization approach, the same upper and lower limit P/E ratio was applied in the selection process of each stock. Consequently, the selection process provided by the $\mathrm{P} / \mathrm{E}$ ratios was rather generic and not tailored to any specific industry. For example, the banking industry P/E ratio historically averages 30 or less whereas in the high tech industry it is not uncommon to see P/E ratios greater than 100 for rapidly growing companies. Further analysis could involve tailoring P/E ratio data to specific industries.

\section{Subjective/Objective}

Given that purely objective criteria were used in the asset selection process, another area of future studies could incorporate subjective criteria. Some possible aspects of subjective criteria that could be incorporated are pubic company news and management's outlook on the company and industry found in financial reports.

\section{Varying Monthly Data Points}

All stock portfolios were optimized using 29 to 36 monthly data points for each stock. All industry portfolios were optimized using exactly 48 monthly data points for each industry. A supplementary analysis could involve varying the amount of data points used during the optimization process to enhance the model. An assumption one could make is that using a greater number of data points may enhance a model's long term performance due to it providing better correlations of past data. Another assumption could be that using a smaller number of data points may enhance a more active portfolio strategy since it focuses more on the short term trends.

\section{Stock Ratings Systems}

Additional analysis could also take into consideration stock ratings systems such as Valueline, MSN Moneycentral Stockscouter, Bloomberg, etc to aid in selecting the most optimal stocks for optimization. Attempts were made to acquire past ratings on stocks from these research firms but do to budget and time constraints and the proprietorship nature of their data it was not possible. Consequently, only several key factors were use in selecting the most optimal stocks for optimization.

\section{Varying Number Of Assets Per Portfolio}

Accompanying analysis could also involve varying the number of assets within a portfolio, thus increasing or decreasing the number of assets per investment period. Targets of three stocks within nine industries were set for each investment period of the two staged approach. Targets of 30 stocks per investment period were set for the ordinary optimization technique. 


\section{Software/Tool Improvements}

Due to the limitations of the software and LCO technique using Excel, roughly 20 stocks were obtained for each investment period in the ordinary optimization technique. A target of roughly 30 stocks per investment period was set and met by the two staged optimization approach. Consequently, comparisons between the two staged optimization approach and the ordinary optimization technique may not be as precise as desired. Additional studies that produce more equivalent stocks per industry between the two models could be pursued.

\section{Rebalancing Frequency}

Given that this work took into account solely a biweekly rebalancing period, the models stand to gain from finding an optimal rebalancing frequency. Further analysis could take into consideration the effects of rebalancing portfolios on a weekly, monthly, bimonthly, or quarterly basis.

\section{Variations Of MVO Techniques}

Further studies could also explore the affects different MVO technique variations such as MVP, CET, BST, LCO, and pooling have on the different investment strategies (ordinary and two staged).

$\underline{\text { Notes }}$ 


\section{$\underline{\text { Notes }}$}

